\title{
Establishment of a rat model with ageing insomnia induced by D-galactosef and para-chlorophenylalanine
}

\author{
XIAO-JUAN REN ${ }^{1,2}$, QING-QUAN WANG ${ }^{1}$, XING-PING ZHANG ${ }^{1}$, \\ GUAN-YING WANG ${ }^{1}$, TAO LIU $^{3}$, NING DENG $^{4}$ and DE-QI YAN ${ }^{1}$ \\ ${ }^{1}$ Department of Internal Medicine, Traditional Chinese Medicine Hospital Affiliated to Xinjiang Medical University, Urumqi, \\ Xinjiang 830000; ${ }^{2}$ Department of Gerontology, Xinjiang Urumqi Municipality Traditional Chinese Medicine Hospital, \\ Affiliated to Xinjiang Medical University, Urumqi, Xinjiang 830002; ${ }^{3}$ Department of Gerontology, Traditional Chinese \\ Medicine Hospital Affiliated to Xinjiang Medical University, Urumqi, Xinjiang 830000; ${ }^{4}$ College of Traditional \\ Chinese Medicine, Xinjiang Medical University, Urumqi, Xinjiang 830011, P.R. China
}

Received November 12, 2019; Accepted June 10, 2020

DOI: $10.3892 /$ etm.2020.9080

\begin{abstract}
The current study aimed to establish a rat model of ageing insomnia induced by D-galactose and/or para-chlorophenylalanine. Following establishment of the model, body weights were measured, and Morris water maze and pentobarbital-induced sleep tests were performed. The serum levels of inflammatory mediators and the neural levels of neurotransmitters were detected. The results demonstrated that the body weights of PCPA+D-gal-induced ageing insomnia rats decreased significantly. Ageing insomnia rats exhibited longer latencies to the platform in the Morris water maze tests and fewer target crossings. The sleep latency of the model rats was longer and sleep time was shorter by contrast. The relative expression of hippocampal IL-6, TNF- $\alpha, \mathrm{NF}-\kappa \mathrm{B}$ and mGluR2 mRNA of the PCPA+D-gal-induced ageing insomnia group was higher, while the relative expression of 5- $\mathrm{HT}_{1 \mathrm{~A}} \mathrm{R}$ and $\mathrm{GABA}_{\mathrm{A}} \mathrm{R}_{\mathrm{a} 1}$ mRNA were lower. The serum levels of IL-1 $\beta$, IL-6, TNF- $\alpha$ and brain level of glutamate increased in the PCPA+D-gal-induced ageing insomnia group, while the levels of 5-HT and GABA decreased. In conclusion, memory function, sleep time, expression of inflammatory factors and neurotransmitters are altered in ageing insomnia rats induced by $\mathrm{D}$-galactose and para-chlorophenylalanine, indicating the successful establishment of a murine model of ageing insomnia.
\end{abstract}

Correspondence to: Dr Xing-Ping Zhang, Department of Internal Medicine, Traditional Chinese Medicine Hospital Affiliated to Xinjiang Medical University, 116 Huanghe Road, Urumqi, Xinjiang 830000, P.R. China

E-mail: xjmyzxp@163.com

Key words: ageing insomnia, rat model, inflammatory factors, neurotransmitters

\section{Introduction}

Insomnia is a subjective experience characterized by difficulty in falling asleep and/or staying asleep, which results in poor sleep quality and/or quantity (1). The sleep characteristics of the elderly are different from those of young people (2). In China, the prevalence of insomnia is $17 \%$ and the percentage of the people whose sleep time is $<6 \mathrm{~h}$ is $23 \%$ (3), with increasing prevalence of insomnia among the elderly (4). The prevalence of elderly insomnia in China is $47.2 \%$ (5) and $57 \%$ in America (6). Insomnia severely affects the physical health and life quality of the elderly (7-11) and increases social and economic burden (6).

The main clinical characteristic of ageing insomnia is difficulty initiating sleep (12). Ageing individuals spend more time in the lighter stages of sleep than in deep sleep in non-rapid eye movement sleep (NREM), as demonstrated by electroencephalography (13). The main treatments of ageing insomnia are psychological/behavioral therapies, pharmacological treatment or a combination of both (6). Pharmacological treatments are initially recommended when non-pharmacological options do not attain satisfying sleep (14). Currently, the commonly used ageing animal models include: D-galactose (D-gal)-induced subacute (15), $\beta$-amyloid induced $(16,17)$, thymic senescence (18), rapid (19) and natural (20) ageing models. The ageing model induced by $\mathrm{D}$-gal has been widely used $(21,22)$ and has been evaluated in behavioral, biochemical and neurochemical aspects (23). The modeling methods of animal models of insomnia mainly include chemical reagent stimulation using intraperitoneal injection of para-chlorophenylalanine (PCPA) (24), the horizontal platform environmental deprivation method (25), the stress stimulation sleep deprivation method (26), the gentle stimulation deprivation method and the forced exercise deprivation method (27). Rat and mouse models can be used; however, rat models are optimal due to model stability in the establishment of the ageing model induced by D-gal (28). Therefore, the current study established rat models.

Inflammatory factors are closely related to sleep and ageing $(29,30)$. Sleep has a regulatory effect on immune 
function, with pro-inflammatory cytokines reaching their peak levels during early night sleep and anti-inflammatory cytokines reaching their peak levels during daytime waking hours (31). Inflammatory cytokines interleukin (IL)-1 and tumor necrosis factor (TNF)- $\alpha$ participate the regulation of physiological sleep in the central nervous system (32). Inflammatory cytokines are also involved in oxidative stress, and inflammation is associated with aging (33). IL-1 $\beta$, IL-6 and TNF- $\alpha$ are the key inflammatory cytokines involved in oxidative stress. They are closely related to reactive oxygen species (ROS) and promote the activation of $\mathrm{NF}-\kappa \mathrm{B}$, as well as the expression of proinflammatory factor-induced nitric oxide synthase and cyclooxygenase-2 (34). Sleep is closely related to neurotransmitters (35). Glutamate, an important excitatory amino acid transmitter in brain tissue, can stimulate the activity of neurons (36). Gamma-aminobutyric acid (GABA), an important inhibitory neurotransmitter in the central nervous system, can inhibit neuronal excitability and exert sedative and hypnotic effects (37). Glutamate and GABA play an important role in maintaining the balance of nerve cell inhibition and excitation function (38).

In the current study, the ageing insomnia rat model was induced by continuous subcutaneous injection of D-gal and intraperitoneal injection of PCPA. The levels of senility and sleep characteristics of ageing insomnia rat model were evaluated, including memory function, sleep duration, inflammation factors and neurotransmitters. The results of the current study may provide experimental evidence for further research on ageing insomnia.

\section{Materials and methods}

Reagents. D-gal (purity, 99\%) was purchased from Beijing Solarbio Science and Technology Co., Ltd. (cat no. 1013G051). PCPA and pentobarbital were purchased from Sigma-Aldrich, Merck KGaA (cat no. SHBJ7057). IL-1 $\beta$ (cat no. JL20884), IL-6 (cat no. JL20896), TNF- $\alpha$ (cat no. JL29364), 5-hydroxytryptamine (5-HT; cat no. JL13043), glutamate (cat no. JL13664) and gamma-aminobutyric acid (GABA; cat no. JL47835) kits were purchased from Shanghai JiangLai Biotech. Co. Ltd. TRIzol ${ }^{\circledR}$ reagent was obtained from Invitrogen (Invitrogen; Thermo Fisher Scientific, Inc.). RevertAid First Strand cDNA Synthesis kit was purchased from Thermo Fisher Scientific, Inc. TB Green Premix Ex Taq II (Tli RNase Plus) was purchased from Takara Bio, Inc.

Animals. A total of 40 male Sprague Dawley rats (weight, $200 \pm 20 \mathrm{~g}$; age, 2 months) were obtained from the Xinjiang Medical University Animal experiment center [approval protocol no. SYXK (X) 2018-003]. The rats were housed in a specific pathogen-free environment with room temperature of $25 \pm 2^{\circ} \mathrm{C}, 12$-h light/dark cycles and free access to water and food. Their health and behavior were monitored daily. The primary humane endpoints used to determine when animals should be euthanized were reduced heart and respiration rate. 'Guidelines for euthanasia of experimental animals' was followed to minimize suffering and distress of the animals (39).

Experimental procedures were conducted in accordance with the China Experimental Animals Administration
Legislation and were approved by the Ethics Committee of Xinjiang Medical University.

Establishment of rat models. Ageing rat model induced by D-gal or by PCPA are two classical rat models used in ageing and insomnia research, respectively $(21,23)$. D-gal and PCPA were used to establish ageing insomnia rat model in the current study, which was a composite rat model. A total of 40 rats were randomly divided into 4 groups: Controls, PCPA group (insomnia group), D-gal group (ageing group) and PCPA+D-gal group (ageing insomnia group) with 10 rats in each group.

Controls were subcutaneously injected with normal saline $(120 \mathrm{mg} / \mathrm{kg})$ for 42 days, then intraperitoneally injected with normal saline $(300 \mathrm{mg} / \mathrm{kg})$ for 3 days. Rats in the PCPA group were subcutaneously injected with normal saline $(120 \mathrm{mg} / \mathrm{kg}$ ) for 42 days, then intraperitoneally injected with PCPA (300 mg/kg) for 3 days. Rats in the D-gal group were subcutaneously injected with D-gal $(120 \mathrm{mg} / \mathrm{kg})$ for 42 days and then intraperitoneally injected with normal saline $(300 \mathrm{mg} / \mathrm{kg}$ ) for 3 days. Rats in the PCPA+D-gal group were subcutaneously injected with D-gal $(120 \mathrm{mg} / \mathrm{kg})$ for 42 days and then intraperitoneally injected with PCPA $(300 \mathrm{mg} / \mathrm{kg})$ once a day for 3 days. Acute and/or adverse reactions of D-gal and PCPA were not observed during the experiment. Weight gain was measured every week and for the final time on the day 46 .

Morris water maze test. The spatial memory of the rats was assessed via the Morris water maze test on day 46. The Morris Water Maze (Chengdu Taimeng Co., Ltd.) consisted of a tank (radius, $120 \mathrm{~cm}$; height, $50 \mathrm{~cm}$ ) containing water at a height of $30 \mathrm{~cm}$ and a temperature of $\sim 25^{\circ} \mathrm{C}$. The water maze was divided into 4 quadrants and the escape platform (diameter, $12 \mathrm{~cm}$ ) was placed at a fixed position in the $3 \mathrm{rd}$ quadrant, $2.5 \mathrm{~cm}$ under the water. The midpoint was selected as the fixed entry point in each quadrant. The rats were placed into the water with their backs to the wall of the pool and detained on the platform for $10 \mathrm{sec}$ as a sign of success in finding the platform. A water maze device was used to record the time required for the rats to find the platform from the entry point, which was defined as escape latency. Rats stayed on the platform for $10 \mathrm{sec}$ and then the next quadrant experiment was conducted. When the rats took $>2$ min to find the target platform, they were directed to the platform, stayed there for $10 \mathrm{sec}$ and escape latency was recorded as $2 \mathrm{~min}$. For spatial navigation training, the experiment was conducted once a day at each water entry point in each quadrant for a total of 4 times for 5 days. It is hypothesized that the time of each rat crossing the original platform within 2 min reflects the spatial memory ability, and the platform was removed only on the 6th day. Latency time to the platform and the number of target crossings were recorded and processed by a computer equipped with a TaiMeng Behavior Analysis System (Chengdu Taimeng Co., Ltd.).

Measurement of pentobarbital-induced sleeping behavior. Experiments were carried out on day 46. Pentobarbital was diluted with $0.9 \%$ physiological saline and intraperitoneally injected into the rats $(35 \mathrm{mg} / \mathrm{kg})$. The rats were then 
placed into another cage. Sleep latency was recorded as time elapsed between the pentobarbital injection and the time that rats could maintain $60 \mathrm{sec}$ without flipping. Sleep time was recorded as the time between the elapse and the time that rats could not continue to remain in the supine position within $30 \mathrm{sec}$.

Sample collection. Following behavioral tests, the rats were anesthetized with $10 \%$ chloral hydrate (Chengdu Kelong Chemical Co., Ltd.) by intraperitoneal injection into the abdominal cavity with $300 \mathrm{mg} / \mathrm{kg}$ (40). No sign of peritonitis was observed following the injection. No rats died during the experiment and all 40 rats were anesthetized. Blood samples $(8-10 \mathrm{ml})$ were collected from the abdominal aorta. The rats died of hemorrhagic shock following blood sample collection. Death was determined by non-spontaneous breathing, lack of heartbeat and cold limbs. Cardiac and respiratory arrest were observed for 3-5 min to confirm death. Body weight at the time of sacrifice (on day 46) are shown in Table SI. Brain tissue was dissected on ice for further analysis. Experiments, including anesthesia of rats, abdominal aortic blood collection, brain tissue separation, plasma centrifugation $\left(2,000 \mathrm{x} \mathrm{g}\right.$ for $15 \mathrm{~min}$ at $\left.4^{\circ} \mathrm{C}\right)$, liquid nitrogen quick-freezing tissue, labeling and preservation of tissue and body disposal lasted $\sim 5 \mathrm{~h}$.

ELISA. Neural serum levels of IL-1 $\beta$, IL-6, TNF- $\alpha$ and neurotransmitter levels of 5-HT, glutamate and in brain tissue were detected using ELISA kits, according to the manufacturer's protocol.

Reverse transcription quantitative (RT-q) PCR. The hippocampi were isolated and collected to examine the mRNA level of inflammatory cytokines and neurotransmitter receptors. RNA extraction was conducted using TRIzol ${ }^{\circledR}$ reagent. cDNA was synthesized using a RevertAid First Strand cDNA Synthesis kit, according to the manufacturers' instructions. RT-qPCR reactions were conducted by a CFX96 RT-qPCR system (Bio-Rad Laboratories, Inc.) using TB Green ${ }^{\mathrm{TM}}$ Premix Ex Taq ${ }^{\mathrm{TM}}$ II (Tli RNase Plus). The thermocycling conditions included pre-denaturation at $95^{\circ} \mathrm{C}$ for $10 \mathrm{~min}$, denaturation at $95^{\circ} \mathrm{C}$ for $10 \mathrm{sec}$, annealing for $10 \mathrm{sec}$ (annealing temperatures: $60^{\circ} \mathrm{C}$ for nuclear factor $\kappa$-light-chain-enhancer of activated $\mathrm{B}$ cells $(\mathrm{NF}-\kappa \mathrm{B}), 5$-hydroxytryptamine $1 \mathrm{~A}$ receptor $\left(5-\mathrm{HT}_{1 \mathrm{~A}} \mathrm{R}\right)$ and metabotropic glutamate receptor 2 (mGluR2); $62^{\circ} \mathrm{C}$ for IL- 6 and GABA receptor $\alpha 1$ subtype $\left(\mathrm{GABA}_{\mathrm{A}} \mathrm{R}_{\alpha 1}\right)$; $62.5^{\circ} \mathrm{C}$ for $\mathrm{TNF}-\alpha$ ), extension at $72^{\circ} \mathrm{C}$ for $10 \mathrm{sec}, 40$ cycles of extension at $95^{\circ} \mathrm{C}$ for $15 \mathrm{sec}$ and extension at $60^{\circ} \mathrm{C}$ for $1 \mathrm{~min}$. Measurements were performed in triplicate. The mRNA level of inflammatory cytokines and neurotransmitters was normalized to reference $G A P D H$ gene and quantified as relative expression of mRNA using the $2^{-\Delta \Delta \mathrm{Cq}}$ method (41). The primer sequences are listed in Table I.

Statistical analysis. Each experiment was repeated three times. Data are presented as the mean \pm standard deviation or standard error. SPSS software (version 21.0; IBM Corp.) was used for statistical analysis. One-way ANOVA and Tukey's post-hoc test were performed to compare differences between groups. $\mathrm{P}<0.05$ was considered to indicate a statistically significant difference.

\section{Results}

Insomnia rats and ageing insomnia rats lost body weight rapidly on day 45 due to sleep deprivation induced by PCPA injection. The body weight of the rats in different groups were initially observed following model establishment. The results demonstrated that rats in the D-gal and PCPA+D-gal groups appeared to shed hair and move slower from the 5th week, while rats in the PCPA and the PCPA+D-gal groups appeared to be excited and irascible from day 43. Weight gain in the D-gal and PCPA+D groups was significantly lower compared with controls on days 7-42 (Fig. 1). Furthermore, weight gain in the PCPA group was significantly higher compared with $\mathrm{PCPA}+\mathrm{D}$ group on days 7-42. Weight gain in the PCPA and PCPA+D-gal groups was significantly lower compared with controls on day 45. Moreover, weight gain in the D-gal group was significantly higher compared with the PCPA+D-gal group on day 45.

Spatial memory ability decreases in ageing insomnia PCPA+D-gal rats. A Morris water maze was performed to assess the spatial memory ability of the rats. Rats in the $\mathrm{PCPA}+\mathrm{D}$-gal groups suffered significant impairment in spatial learning ability on account of the longer latency time compared with controls from days 2-5 (Fig. 2A). Furthermore, escape latency in the PCPA group was significantly shorter compared with the PCPA+D-gal group from days 2-5 (Fig. 2A). No significant difference of escape latency was found between the ageing D-gal and insomnia PCPA groups (Fig. 2A).

Subsequently, the spatial memory was evaluated on the 6th day. The escape latency of rats in PCPA+D-gal groups was longer compared with the control group (Fig. 2B). Furthermore, escape latency in the D-gal and PCPA groups was shorter compared with the PCPA+D-gal group (Fig. 2B).

Rats in D-gal and PCPA+D-gal groups exhibited lower target crossing numbers compared with the control group (Fig. 2C). Additionally, there were significantly more target crossings in the PCPA group compared with the PCP+D-gal group (Fig. 2C). No significant difference was found between the PCPA and D-gal groups (Fig. 2C).

According to the results of Morris water maze tests, the current study concluded that the spatial memory ability of the PCPA+D-gal ageing insomnia rats significantly decreased compared with the control group.

Ageing insomnia PCPA+D-gal rats exhibit shorter sleep latency and sleep time. Sleep latency and sleep time through pentobarbital injections was investigated. The results demonstrated that sleep latency of PCPA and PCPA+D-gal rats was significantly longer compared with the control group (Fig. 3A). Furthermore, D-gal rats exhibited shorter sleep latency compared with the PCPA+D-gal group (Fig. 3A).

The sleep time of D-gal and PCPA+D-gal rats was significantly shorter compared with the control group (Fig. 3B). Additionally, PCPA rats exhibited longer sleep time compared with the PCPA+D-gal group (Fig. 3B). There was no significant difference between the PCPA and D-gal groups (Fig. 3B). 
Table I. Forward and reverse primers used for reverse transcription quantitative PCR analysis.

\begin{tabular}{|c|c|c|}
\hline Primer & Forward & Reverse \\
\hline IL-6 & 5'-AGGAGTGGCTAAGGACCAAGACC-3' & 5'-TGCCGAGTAGACCTCATAGTGACC-3' \\
\hline TNF- $\alpha$ & 5'-CCAATGGCGTGGAGCTGAGAG-3' & 5'-TCTGGTAGGAGACGGCGATGC-3' \\
\hline $\mathrm{NF}-\kappa \mathrm{b}$ & 5'-TGTGGTGGAGGACTTGCTGAG G-3' & 5'-AGTGCTGCCTTGCTGTTCTTGAG-3' \\
\hline $5-\mathrm{HT}_{1 \mathrm{~A}} \mathrm{R}$ & 5'-AGGACCACGGCTACACCATCTAC-3' & 5'-CTGACAGTCTTGCGGATTCGGAAG-3' \\
\hline mGluR2 & 5'-ATC ACTGGTGGTATTGGCGGTTCC-3' & 5'-TGGCACTGGTAGAGGCGTAGC-3' \\
\hline $\mathrm{GABA}_{\mathrm{A}} \mathrm{R}_{\alpha 1}$ & 5'-TGAGCACACTGACTGGAAGAAGC-3' & 5'-TGGTCTCAGGCGATTGTCATAACC-3' \\
\hline GAPDH & 5'-GACATGCCGCCTGGAGAAAC-3' & 5'-AGCCCAGGATGCCCTTTAGT-3' \\
\hline
\end{tabular}

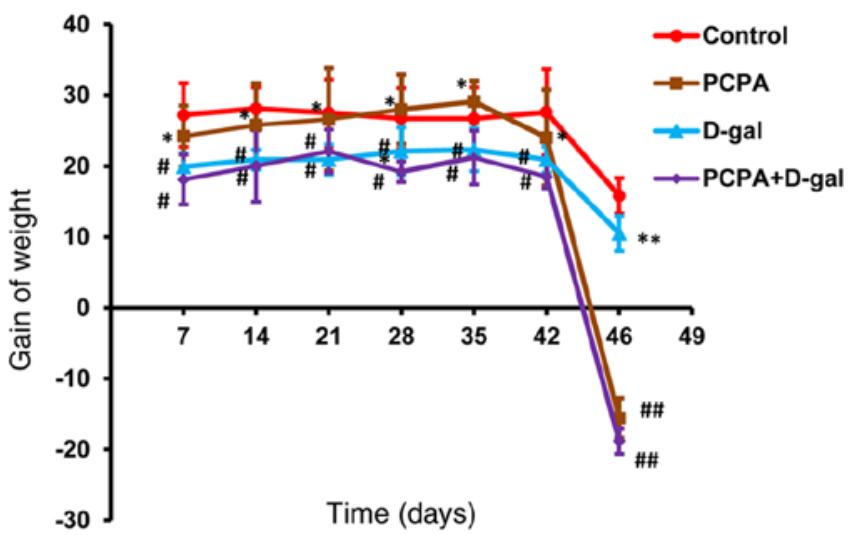

Figure 1. Weight gain in rats was measured each week and for the final time on day 46. Data are presented as mean \pm standard deviation. ${ }^{\#} \mathrm{P}<0.05$ and ${ }^{\# \#} \mathrm{P}<0.01$ vs. control group. ${ }^{*} \mathrm{P}<0.05$ and ${ }^{* *} \mathrm{P}<0.01$ vs. $\mathrm{PCPA}+\mathrm{D}$-gal group. PCPA, para-chlorophenylalanine; D-gal, D-galactose.

Expression of pro-inflammatory cytokines and neurotransmitter receptors at $m R N A$ level are also influenced by $D$-gal and PCPA injections. Hippocampal IL-6, TNF- $\alpha$, NF- $\kappa$ B, 5-HT ${ }_{1 A} \mathrm{R}$, mGluR2 and GABA ${ }_{A} \mathrm{R}_{\alpha 1}$ mRNA expression was determined using RT-qPCR to establish whether inflammatory cytokines and neurotransmitter receptors were involved in cognitive impairment and insomnia. The results demonstrated that IL- 6 , TNF- $\alpha$ and NF- $\kappa$ B mRNA expression were upregulated in the hippocampus of rats in the PCPA, D-gal and PCPA+D-gal groups compared with the control group (Fig. 4A-C). However, there was no significant difference in the IL-6, TNF- $\alpha$ and NF- $\kappa$ B mRNA expression levels in the PCPA and D-gal groups compared with the PCPA+D-gal group. Additionally, the PCPA and PCPA+D-gal groups demonstrated downregulated 5- $\mathrm{HT}_{1 \mathrm{~A}} \mathrm{R}$ mRNA expression compared with the control group (Fig. 4D). The PCPA, D-gal and PCPA+D-gal groups demonstrated downregulated $\mathrm{GABA}_{\mathrm{A}} \mathrm{R}_{\alpha 1}$ mRNA expression compared with the control group (Fig. 4F). No significant difference was discovered between D-gal, PCPA and PCPA+D-gal groups. Furthermore, the PCPA and PCPA+D-gal groups exhibited upregulated mGluR2 mRNA expression compared with the control group (Fig. 4E). The D-gal group also demonstrated downregulated mGluR2 mRNA expression compared with the PCPA+D-gal group. The results indicate that the subcutaneous injection of D-gal, intraperitoneal injection of PCPA and combined injection of D-gal and PCPA altered inflammatory cytokine and neurotransmitter receptor mRNA expression in the rat hippocampus.

Expression levels of pro-inflammatory cytokines in neural serum and neurotransmitters in neural tissue. Subsequently, ELISA assays were used to investigate the expression of pro-inflammatory cytokines and neurotransmitters. Serum expression of IL-1 $\beta$, IL- 6 and TNF- $\alpha$ in the PCPA+D-gal rats were significantly increased compared with the control group (Fig. 5A-C). Furthermore, the levels of these cytokines in the PCPA and D-gal rats were significantly decreased compared with the PCPA+D-gal group. No significant difference of IL-1 $\beta$, IL- 6 and TNF- $\alpha$ was found between the PCPA and D-gal groups. The results indicated that the subcutaneous injection of D-gal, intraperitoneal injection of PCPA and combined injection of D-gal and PCPA all induced significant chronic inflammation in the rats.

Furthermore, neural levels of 5-HT, glutamate and GABA were measured. The results demonstrated a significant decrease in the levels of 5-HT in the PCPA+D-gal groups compared with the control group (Fig. 5D). The levels of 5-HT in brains of D-gal and PCPA rats were significantly higher compared with the PCPA+D-gal group. The PCPA+D-gal groups exhibited a significant increase in the levels of glutamate compared with the control group (Fig. 5E). Furthermore, neural glutamate levels in the D-gal and PCPA rats were significantly decreased compared with the PCPA+D-gal group (Fig. 5E). The PCPA and $\mathrm{PCPA}+\mathrm{D}$-gal groups exhibited a significant decrease in GABA levels compared with the control group (Fig. 5F). No significant difference was found in the levels of 5-HT, glutamate and GABA between the D-gal and PCPA groups. These results demonstrated that the subcutaneous injection of D-gal, intraperitoneal injection of PCPA and the combined injection of D-gal and PCPA induced expression changes of the neurotransmitters in brains of the rats.

\section{Discussion}

Oxidative damage induced by ROS serves a crucial role in the pathophysiology of ageing $(42,43)$. High doses of D-gal induce ROS overexpression via the metabolism of D-gal (44). The injection of D-gal results in the increase of oxygen free radicals in the rat brain (21). Additionally, ageing alterations induced by D-gal are similar to natural ageing processes (45). A previous study demonstrated that the levels of neurotransmitters in 
A
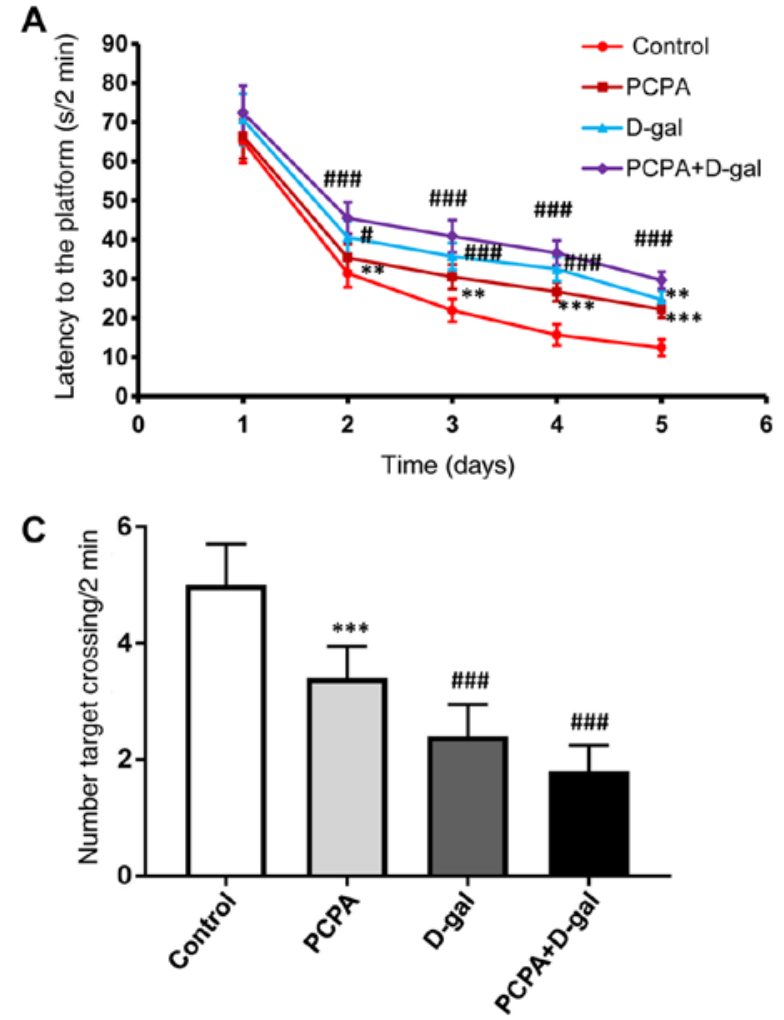

B

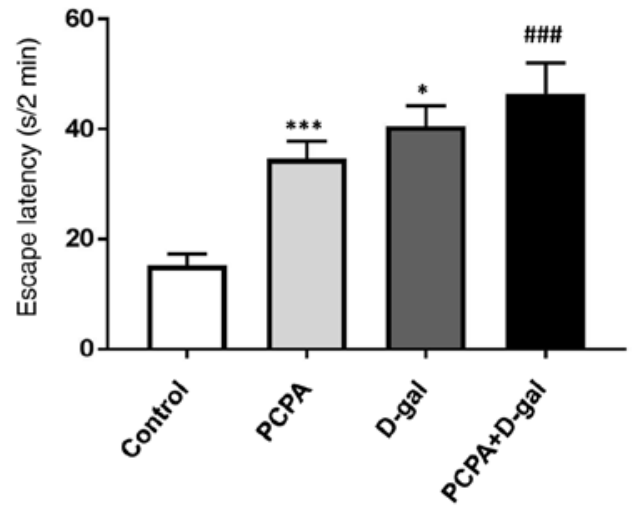

Figure 2. The spatial memory ability of rats was observed using a Morris water maze test. (A) Latency to the platform within 2 min during 5 days spatial navigation training ( $/ 2$ min). (B) Escape latency to the platform within 2 min on day 6 ( $/ 2$ min). (C) The number of target crossings within 2 min. Data are presented as mean \pm SEM. ${ }^{\#} \mathrm{P}<0.05$ and ${ }^{\# \# \#} \mathrm{P}<0.001$ vs. control group. ${ }^{*} \mathrm{P}<0.05,{ }^{* *} \mathrm{P}<0.01$ and ${ }^{* * *} \mathrm{P}<0.001$ vs. PCPA+D-gal group. PCPA, para-chlorophenylalanine; D-gal, D-galactose.
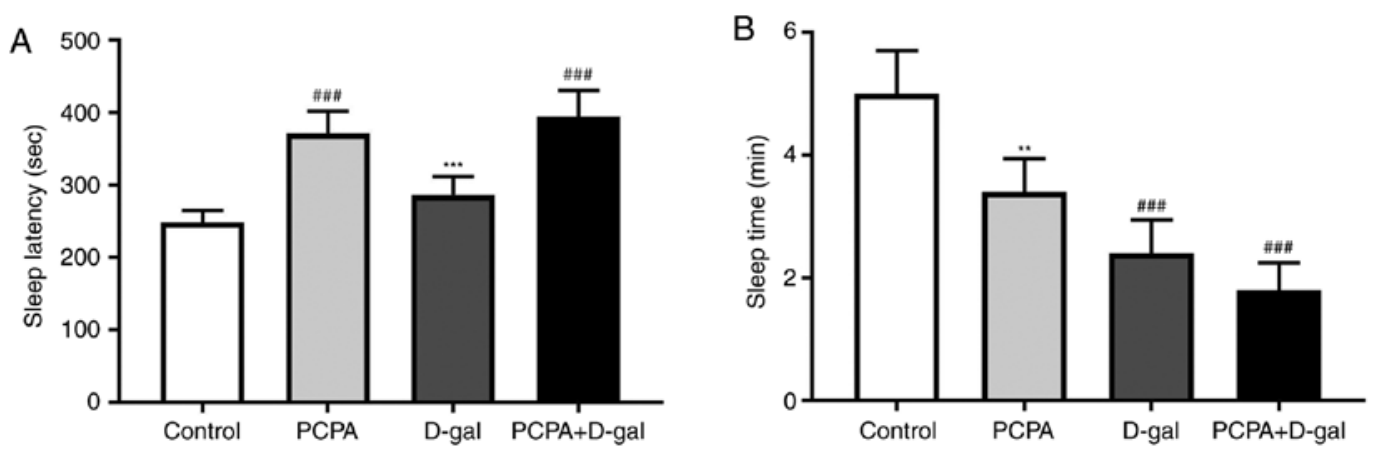

Figure 3. Pentobarbital (35 mg/kg) was intraperitoneally injected into rats to observe sleep latency and sleep time. The (A) sleep latency and (B) sleep time were recorded. Data are presented as mean \pm SEM. ${ }^{\# \# \#} \mathrm{P}<0.001$ vs. control group. ${ }^{* *} \mathrm{P}<0.01$ and ${ }^{* * *} \mathrm{P}<0.001$ vs. PCPA+D-gal group. PCPA, para-chlorophenylalanine; D-gal, D-galactose.

the brain of PCPA-induced insomnia rats were altered (23). Therefore, D-gal and PCPA were used in this study to establish rat models of ageing and insomnia, respectively. Furthermore, another previous study reported that $\mathrm{D}$-gal-induced ageing rats lose weight rapidly compared with the control group (46). In the current study, the results indicated that rats in both D-gal and PCPA+D-gal groups lost weight rapidly compared with control rats.

Cognitive decline increases with age during natural ageing $(47,48)$. It has been reported that in the Morris water maze, the latency time of D-gal rats was longer, the number of target crossings was lower and that cognitive function decreased (49). Sleep disorder is closely related to cognition (50) and patients with primary insomnia have been reported to suffer from subjective memory impairment in virtual water mazes (51). Sleep duration is negatively correlated with age in older adults (52) and chronic insomnia in the elderly is likely to exacerbate cognitive impairment (53). In the current study, D-gal-induced ageing rats, PCPA-induced the insomnia rats and D-gal- and PCPA-induced ageing insomnia rats demonstrated cognitive decline in the Morris water maze. Therefore, the ageing and insomnia model exhibited decreased cognitive function.

Chronic inflammation is closely related to ageing (54). Inflammatory factors, such as IL- $1 \beta$, IL-6 and TNF- $\alpha$, serve roles in D-gal-induced oxidative stress to simulate the 
A
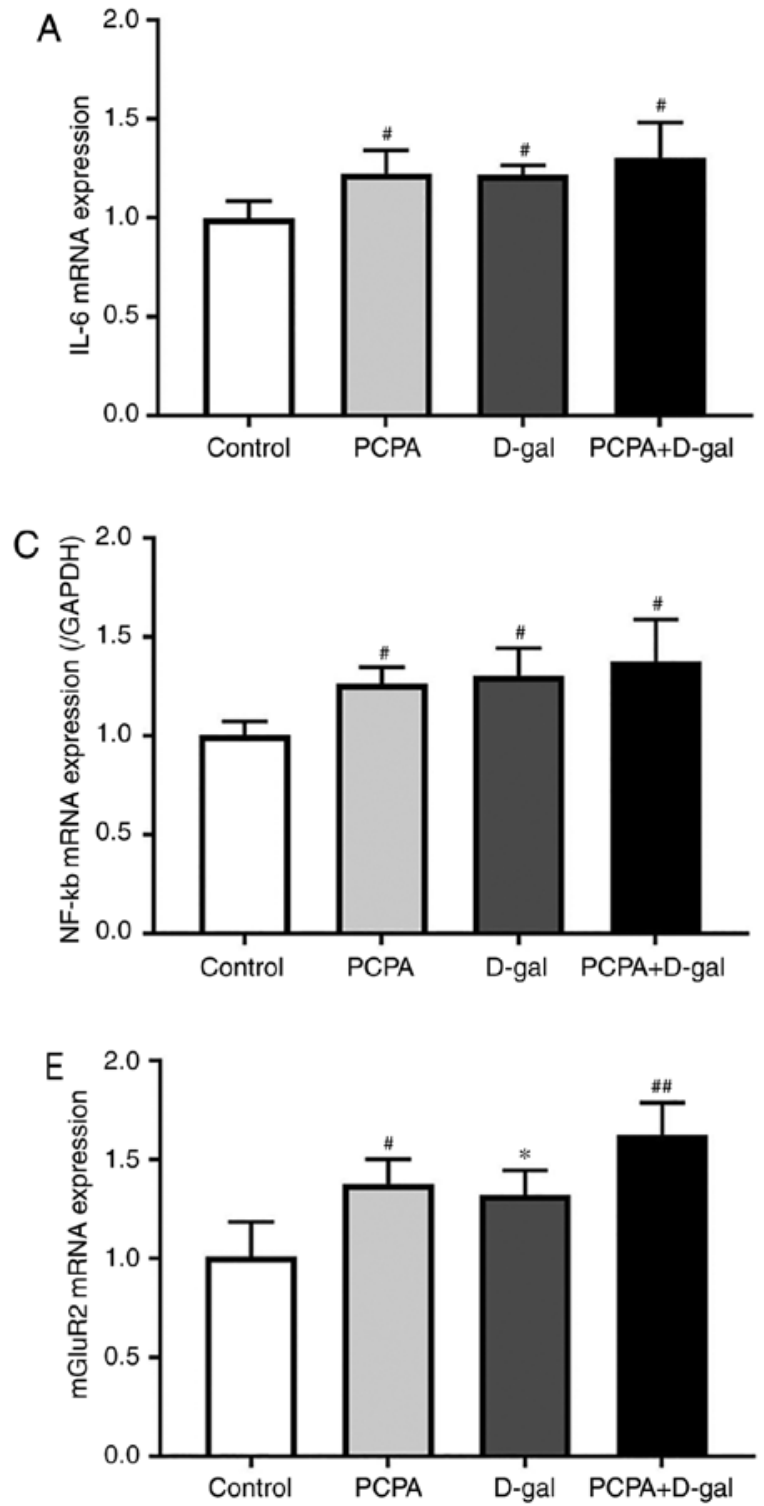

B
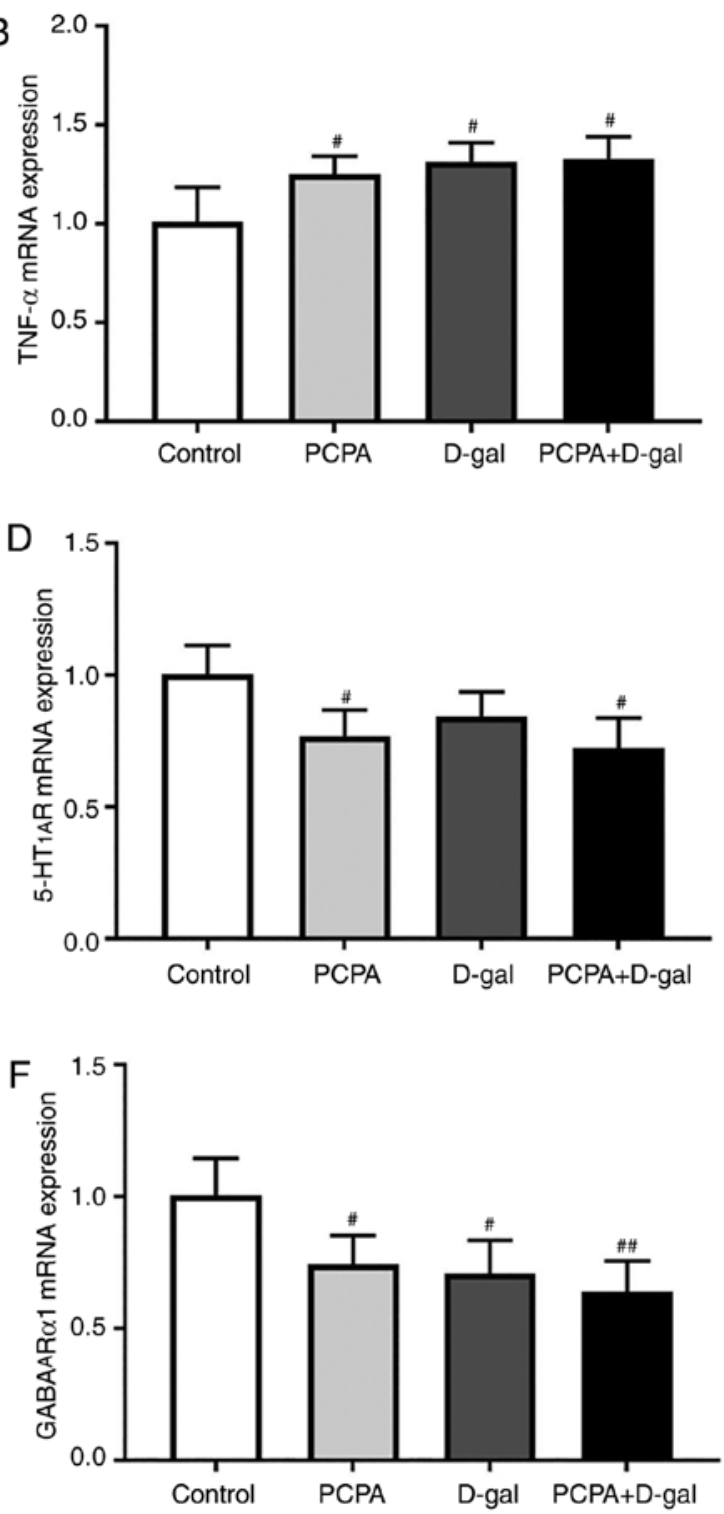

Figure 4. Gene expression of inflammatory factors IL-6, TNF- $\alpha$, NF- $k b$ and neurotransmitter receptors of 5-HT ${ }_{1 A} \mathrm{R}$, $\mathrm{mGluR}_{2}$, Gamma-aminobutyric acid A receptor $\alpha 1$ subtype in the hippocampus were assayed using RT-PCR. mRNA expression of (A) IL-6, (B) TNF- $\alpha$, (C) NF-kb, (D) 5-HT 1 R, (E) mGluR2 mRNA, and (F) GABA $R_{A}$. Data are presented as mean \pm SEM. ${ }^{\#} \mathrm{P}<0.05,{ }^{\# \#} \mathrm{P}<0.01$ vs. control group. ${ }^{*} \mathrm{P}<0.05$ vs. PCPA+D-gal group. IL-6, interleukin 6; TNF- $\alpha$, tumor necrosis factor $\alpha$; NF- $k$ b, nuclear factor $\kappa$-light-chain-enhancer of activated B cells; 5-HT ${ }_{1 A} \mathrm{R}, 5$-hydroxytryptamine $1 \mathrm{~A}$ receptor; mGluR2, metabotropic glutamate receptor 2 .

ageing process (46). It has been reported that the plasma concentrations of IL-1 $\beta$, IL- 6 and TNF- $\alpha$ in the D-gal and PCPA induced ageing insomnia rats were significantly higher compared with the control group (55). Furthermore, inflammatory cytokines affect neurotransmitters related to sleep, such as norepinephrine and 5-HT, which are transmitted to the central nervous system $(56,57)$. IL-1 $\beta$, IL- 6 and TNF- $\alpha$ are the key inflammatory factors related to sleep regulation (58), and IL-1 $\beta$ increases non-rapid eye movement sleep in electroencephalography (59). The $\mathrm{I}-\kappa / N F-\kappa \mathrm{B}$ signaling pathway is associated with sleep regulation and the immune system and hippocampus play a central role in insomnia (60). A previous study demonstrated that plasma levels of IL- 6 and TNF- $\alpha$ in PCPA-induced insomnia rats were significantly higher compared with the control group (25). In the current study, the results demonstrated that the serum content of IL-1 $\beta$, IL- 6 and
TNF- $\alpha$ and mRNA expression of IL-6, TNF- $\alpha$ and NF- $\kappa$ B in neural tissues in the PCPA+D-gal group were higher compared with control rats.

Glutamate is an important excitatory amino acid transmitter in brain tissue (36). mGluR2 is distributed in both pre- and post-synaptic neurons, inhibits adenylate cyclization and regulates ion channel receptors by coupling with Gi/o, and negatively regulates neurotransmitter release (61). mGluR2 is involved in the physiology of sleep (62). Additionally, GABA is a major inhibitory neurotransmitter in the central nervous system (37). GABA inhibits neuronal excitation in the nervous system, and GABA receptors are widely used in the treatment of anxiety disorder, insomnia, epilepsy (62). Glutamate and GABA serve important roles in maintaining the stability of the balance between the inhibitory and excitatory functions of nerve cells (63). It has been demonstrated the amount of GABA 

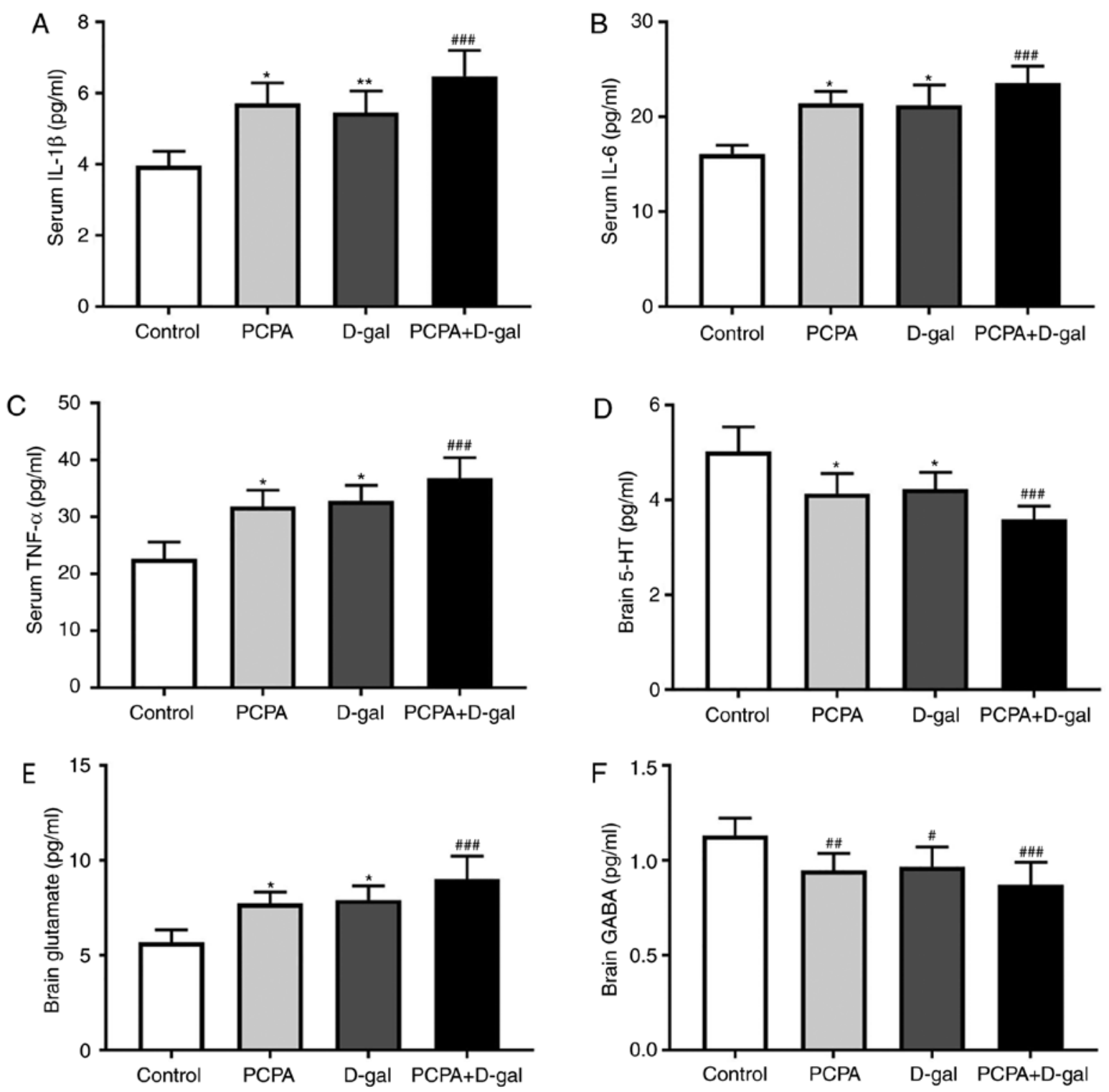

Figure 5. Protein expression of inflammatory factors IL-1 $\beta$, IL-6, TNF- $\alpha$ in serum and expression of 5-HT, glutamate, GABA in neural tissue were assayed via ELISA. Serum (A) IL-1 $\beta$, (B) IL-6 (C), TNF- $\alpha$ and neural (D) 5-HT, (E) glutamate and (F) GABA. Data are presented as mean \pm SEM. ${ }^{\# P}<0.05$, ${ }^{\# \#} \mathrm{P}<0.01$ and

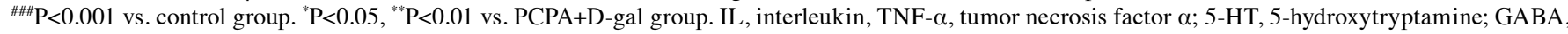
gamma-aminobutyric acid; PCPA, para-chlorophenylalanine; D-gal, D-galactose.

in the brain tissue is positively associated with the changes in sleep-wake depth (64). Furthermore, GABA serves inhibitory roles by binding to its receptor (65) and $\mathrm{GABA}_{\mathrm{A}} \mathrm{R}_{\alpha 1}$ has a sedative effect (66). It has been reported that the expression of glutamate in cerebral cortex, hypothalamus and hippocampus of in PCPA-induced insomnia rats increased, while the content of 5-HT and GABA decreased compared with the control group (23). Woo et al (67) found that the expression of $\mathrm{GABA}_{\mathrm{A}} \mathrm{R}_{\alpha 1}$ protein was upregulated in the hypothalamus of mice with improved sleep time. In the current study, the results demonstrated that expression of 5-HT and GABA decreased while glutamate increased in the D-gal- and PCPA-induced ageing insomnia rats compared with the control group. This indicated that ageing insomnia may be associated with 5-HT, glutamate and GABA neurotransmitters.

The present study established an ageing insomnia rat model by injection of D-galactose and PCPA. Changes in memory ability, inflammatory factors and neurotransmitters of model rats were observed. Future studies should include more behavioral tests, age-related redox marker detections (oxidative stress), apoptotic proteins and body weight/5-HT measurements in order to verify the results of the present study and elucidate ageing insomnia in the rat model.

In conclusion, the current study established an ageing insomnia rat model by an injection combination of D-gal with PCPA, and evaluated the changes in cognitive behavior, sleep duration, inflammation factors and neurotransmitters in $\mathrm{PCPA}+\mathrm{D}$-gal ageing insomnia rats. This ageing insomnia rat model induced by D-gal and PCPA may an provide experimental model for further research on ageing insomnia.

\section{Acknowledgements}

Not applicable. 


\section{Funding}

The present study was supported by the National Natural Science Foundation of China (grant nos. 81560762 and 81960837), the Xinjiang Uygur Autonomous Region Healthy Young Medical Science and Technology Talents Special Research Project (grant no. WJWY-201919), General Project of the Natural Science Foundation of Xinjiang Uygur Autonomous Region (grant no. 2020D01A32) and Xinjiang Uygur Autonomous Region 13th Five-Year Key Discipline (grant no. 1005).

\section{Availability of data and materials}

The datasets used and/or analysed during the present study are available from the corresponding author on reasonable request.

\section{Authors' contributions}

XR, QW, XZ and ND designed the study; XZ supplied the samples for this study; XR, GW, TL and DY analyzed the data; $\mathrm{XZ}$ supervised the whole study; XR and XZ wrote he manuscript. All authors read and approved the final manuscript.

\section{Ethics approval and consent to participate}

All experimental procedures were conducted in accordance with China Experimental Animals Administration Legislation and were approved by the Ethics Committee of Xinjiang Medical University (Urumqi, China).

\section{Patient consent for publication}

Not applicable.

\section{Competing interests}

The authors declare that they have no competing interests.

\section{References}

1. Chinese guidelines for diagnosis and treatment of insomnia in adults (2017 version). Chin J Neurol 51: 324-335, 2018.

2. Qaseemg A, Kansagara D, Forciea MA, Cooke M and Denberg TD; Clinical Guidelines Committee of the American College of Physicians: Management of Chronic insomnia disorder in adults: A clinical practice guideline from the American college of physicians. Ann Intern Med 165: 125-133, 2016.

3. Chen YP, Kartsonaki C, Clarke R, Guo Y, Yu C, Bian Z, Jiang Q, $\mathrm{Li}$ S, Chen J, Li L, et al: Characteristics and correlates of sleep duration, daytime napping, snoring and insomnia symptoms among 0.5 million Chinese men and women. Sleep Med 44: 67-75, 2018.

4. Wang YM, Song M, Wang R, Shi L, He J, Fan TT, Chen WH, Wang L, Yu LL, Gao YY, et al: Insomnia and multimorbidity in the community elderly in China. J Clin Sleep Med 13: 591-597, 2017.

5. Liu Y, Dong YH, Li XY, et al: Meta-analysis of the prevalence of sleep disorder among Chinese elderly aged 60 years and over. Modern Prev Med, 2014

6. Abad VC and Guilleminault C: Insomnia in elderly patients: Recommendations for pharmacological management. Drugs Aging 35: 791-817, 2018.

7. Gulia KK and Kumar VM: Sleep disorders in the elderly: A growing challenge. Psychogeriatrics 18: 155-165, 2018.

8. Kim WH, Kim JH, Kim BS, Chang SM, Lee DW, Cho MJ and Bae JN: The role of depression in the insomnia of people with subjective memory impairment, mild cognitive impairment, and dementia in a community sample of elderly individuals in South Korea. Int Psychogeriatr 29: 653-661, 2017.
9. Leblanc MF, Desjardins S and Desgagne A: Sleep cognitions associated with anxiety and depression in the elderly. Clin Interv Aging 10: 575-582, 2015.

10. Nagai M, Hoshide S, Nishikawa M, Shimada K and Kario K: Sleep duration and insomnia in the elderly: Associations with blood pressure variability and carotid artery remodeling. Am J Hypertens 26: 981-989, 2013.

11. Zhuang J, Zhan Y, Zhang F, Tang Z, Wang J, Sun Y, Ding R, Hu D and Yu J: Self-reported insomnia and coronary heart disease in the elderly. Clin Exp Hypertens 38: 51-55, 2016.

12. Ahmed AE, Al-Jahdali H, Fatani A, Al-Rouqi K, Al-Jahdali F, Al-Harbi A, Baharoon S, Ali YZ, Khan M and Rumayyan A: The effects of age and gender on the prevalence of insomnia in a sample of the Saudi population. Ethn Health 22: 285-294, 2017.

13. Moraes W, Piovezan R, Poyares D, Bittencourt LR, Santos-Silva R and Tufik S: Effects of aging on sleep structure throughout adulthood: A population-based study. Sleep Med 15: 401-409, 2014.

14. Sateia MJ, Buysse DJ, Krystal AD, Neubauer DN and Heald JL: Clinical practice guideline for the pharmacologic treatment of chronic insomnia in adults: An American academy of sleep medicine clinical practice guideline. J Clin Sleep Med 13: 307-349, 2017.

15. Haider S, Liaquat L, Shahzad S, Sadir S, Madiha S, Batool Z, Tabassum S, Saleem S, Naqvi F and Perveen T: A high dose of short term exogenous D-galactose administration in young male rats produces symptoms simulating the natural aging process. Life Sci 124: 110-119, 2015.

16. Liu L, Zhao YH, Zeng CQ and Zeng Y: Research progress in pharmacological effects of Uncaria Hook on Alzheimer disease models. Yao Xue Xue Bao 51: 536-542, 2016 (In Chinese).

17. Wang G, Chen L, Pan X, Chen J, Wang L, Wang W, Cheng R, Wu F, Feng X, Yu Y, et al: The effect of resveratrol on beta amyloid-induced memory impairment involves inhibition of phosphodiesterase-4 related signaling. Oncotarget 7: 17380-17392, 2016.

18. Novoseletskaya AV, Kiseleva NM, Zimina IV, Bystrova OV, Belova OV, Inozemtsev AN, Arion VY and Sergienko VI: Thymus polypeptide preparation tactivin restores learning and memory in thymectomied rats. Bull Exp Biol Med 159: 623-625, 2015.

19. Sasaki K, Han J, Shimozono H, Villareal MO and Isoda H: Caffeoylquinic acid-rich purple sweet potato extract, with or without anthocyanin, imparts neuroprotection and contributes to the improvement of spatial learning and memory of SAMP8 mouse. J Agric Food Chem 61: 5037-5045, 2013.

20. Salazar C, Valdivia G, Ardiles ÁO, Ewer J and Palacios AG: Genetic variants associated with neurodegenerative Alzheimer disease in natural models. Biol Res 49: 14, 2016.

21. Chang YM, Tamilselvi S, Lin HJ, Tsai CC, Lin YM, Day $\mathrm{CH}$, Viswanadha VP, Chang HN, Kuo WW and Huang CY: Alpinia oxyphylla Miq extract ameliorates cardiac fibrosis associated with D-galactose induced aging in rats. Environ Toxicol 34: 172-178, 2019.

22. Li G, Yu J, Zhang L, Wang Y, Wang C and Chen Q: Onjisaponin B prevents cognitive impairment in a rat model of D-galactose-induced aging. Biomed Pharmacother 99: 113-120, 2018.

23. Zhao FF, Zhou YZ, Gao L, Qin XM and Du GH: Advances in the study of the rat model of aging induced by D-galactose. Yao Xue Xue Bao 52: 347-354, 2017 (In Chinese).

24. Bo A, Si L, Wang Y, Bao L and Yuan H: Mechanism of Mongolian medical warm acupuncture in treating insomnia by regulating miR-101a in rats with insomnia. Exp Ther Med 14: 289-297, 2017.

25. Hu Y, Wang YN, Zhang GQ, Dong XZ, Liu WW and Liu P: Gan-Dan-Liang-Yi-Tang alleviates p-chlorophenylalanine-induced insomnia through modification of the serotonergic and immune system. Exp Ther Med 12: 3087-3092, 2016.

26. Zhang B, Zhang Q and Ma YN: Effect of chronic restraint stress on rats' sleep phase and intervention of suanzaoren decoction. Inf Traditional Chin Med 31: 126-129, 2014.

27. Leenaars CH, Dematteis M, Joosten RN, Eggels L, Sandberg H, Schirris M, Feenstra MG and Van Someren EJ: A new automated method for rat sleep deprivation with minimal confounding effects on corticosterone and locomotor activity. J Neurosci Methods 196: 107-117, 2011.

28. Zhao FF, Zhou YZ and Gao L: Research progress of d-galactose induced aging rat model. Chin J Pharm 52: 347-354, 2017.

29. Kang WS, Park HJ, Chuang JH and Kim JW: REM sleep deprivation increases deprivation the expression of interleukin genes in mice hypothalamus. Neuorosci Lett 556: 73-78, 2013. 
30. Li J, Zhang YC and Chen G: Effect of ginkgo biloba extract EGb761 on hippocampal neuronal injury and carbonyl stress of D-Gal-Induced aging rats. Evid Based Complement Alternat Med 2019: 5165910, 2019.

31. Ganz FD: Sleep and immune function. Crit Care Nurse 32: e19-e25, 2012.

32. Mullington JM, Simpson NS, Meier-Ewert HK and Haack M: Sleep loss and inflammation. Best Pract Res Clin Endocrinol Metab 24: 775-784, 2010.

33. Sun L, Zhao Q, Xiao Y, Liu X, Li Y, Zhang J, Pan J and Zhang Z: Trehalose targets Nrf2 signal to alleviate d-galactose induced aging and improve behavioral ability. Biochem Biophys Res Commun 521: 113-119, 2020.

34. Hritcu L, Bagci E, Aydin E and Mihasan M: Antiamnesic and antioxidants effects of ferulagoangulata, essential oil against scopolamine-induced memory impairment in laboratory rats. Neurochem Res 40: 1799-1809, 2015.

35. Wang $\mathrm{W}$ and $\mathrm{Xu} \mathrm{TL}$ : Chloride homeostasis differentially affects $\mathrm{GABA}(\mathrm{A})$ receptor-and glycine receptor-mediated effects on spontaneous circuit activity in hippocampal cell culture. Neurosci Lett 406: 11-16, 2006.

36. Shi YF and Yu YQ: The roles of glutamate in sleep and wakefulness. Zhejiang Da Xue Xue Bao Yi Xue Ban 42: 583-590, 2013 (In Chinese).

37. Jembrek MJ and Vlainic J: GABA receptors: Pharmacological potential and pitfalls. Curr Pharm Des 21: 4943-4959, 2015.

38. He B, Bi K, Jia Y, Wang J, Lv C, Liu R, Zhao L, Xu H, Chen X and $\mathrm{Li}$ Q: Rapid analysis of neurotransmitters in rat brain using ultra-fast liquid chromatography and tandem mass spectrometry: Application to a comparative study in normal and insomnic rats. J Mass Spectrom 48: 969-978, 2013.

39. Cicero L, Fazzotta S, Palumbo VD, Cassata G and Lo Monte AI: Anesthesia protocols in laboratory animals used for scientific purposes. Acta Biomed 89: 337-342, 2018.

40. Zeng X, Zhang L, Sun L, Zhang D, Zhao H, Jia J and Wang W: Recovery from rat sciatic nerve injury in vivo through the use of differentiated MDSCs in vitro. Exp Ther Med 5: 193-196, 2013.

41. Livak KJ and Schmittgen TD: Analysis of relative gene expression data using real-time quantitative PCR and the 2(-Delta Delta $\mathrm{C}(\mathrm{T})$ ) method. Methods 25: 402-408, 2001.

42. Yang C, Du YK, Wang J, Luan P, Yang QL, Huang WH and Yuan L: Transplanted Adipose-derived stem cells ameliorate testicular dysfunction in a D-galactose-induced aging rat model J Cell Physiol 230: 2403-2414, 2015.

43. Zhen YZ, Lin YJ, Li KJ,Zhang GL, Zhao YF, Wang MM, Wei JB, Wei $\mathrm{J}$ and $\mathrm{Hu} \mathrm{G}$ : Effects of rhein lysinate on D-galactose-induced aging mice. Exp Ther Med 11: 303-308, 2016.

44. Qu Z, Zhang J, Yang H, Huo L, Gao J, Chen $\mathrm{H}$ and Gao W: Protective effect of tetrahydropalmatine against d-galactose induced memory impairment in rat. Physiol Behav 154: 114-125, 2016.

45. Cardoso A, Magano S, Marrana F and Andrade JP: D-Galactose high-dose administration failed to induce accelerated aging changes in neurogenesis, anxiety, and spatial memory on young male wistar rats. Rejuvenation Res 18: 497-507, 2015.

46. Chen P, Chen F and Zhou B: Antioxidative, anti-inflammatory and anti-apoptotic effects of ellagic acid in liver and brain of rats treated by D-galactose. Sci Rep 8: 1465, 2018.

47. Bubbico G, Chiacchiaretta P, Parenti M, di Marco M, Panara V, Sepede G, Ferretti A and Perrucci MG: Effects of second language learning on the plastic aging brain: Functional connectivity, cognitive decline, and reorganization. Front Neurosci 13: 423, 2019.

48. Koen JD and Rugg MD: Neural dedifferentiation in the aging brain. Trends Cogn Sci 23: 547-559, 2019.

49. Zhu J, Mu X, Zeng J, Xu C, Liu J, Zhang M, Li C, Chen J, Li T and Wang Y: Ginsenoside Rg1 prevents cognitive impairment and hippocampus senescence in a rat model of D-galactose-induced aging. PLoS One 9: e101291, 2014.

50. Sindi S, Johansson L, Skoog J, Mattsson AD, Sjöberg L, Wang HX, Fratiglioni L, Kulmala J, Soininen H, Solomon A, et al: Sleep disturbances and later cognitive status: A multi-centre study. Sleep Med 52: 26-33, 2018.
51. Kuhn M, Hertenstein E, Feige B, Landmann N, Spiegelhalder K, Baglioni C, Hemmerling J, Durand D, Frase L, Klöppel S, et al: Declarative virtual water maze learning and emotional fear conditioning in primary insomnia. J Sleep Res 27: e12693, 2018.

52. Dzierzewski JM, Dautovich N and Ravyts S: Sleep and cognition in older adults. Sleep Med Clin 13: 93-106, 2018.

53. Porter VR, Buxton WG and Avidan AY: Sleep, cognition and dementia. Curr Psychiatry Rep 17: 97, 2015.

54. Jurk D, Wilson C, Passos JF, Oakley F, Correia-Melo C, Greaves L, Saretzki G, Fox C, Lawless C, Anderson R, et al: Chronic inflammation induces telomere dysfunction and accelerates ageing in mice. Nat Commun 2: 4172, 2014.

55. Duan DD, Wang KX, Zhou YZ, Qin XM, Gao L and Du GH: Baicalein exerts beneficial effects in d-galactose-induced aging rats through attenuation of inflammation and metabolic dysfunction. Rejuvenation Res 20: 506-516, 2017.

56. Ruiz FS, Andersen ML, Martins RC, Zager A, Lopes JD and Tufik S: Immune alterations after selective rapid eye movement or total sleep deprivation in healthy male volunteers. Innate Immun 18: 44-54, 2012.

57. Zhao Q, Peng C, Wu X, Chen Y, Wang C and You Z: Maternal sleep deprivation inhibits hippocampal neurogenesis associated with inflammatory response in young offspring rats. Neurobiol Dis 68: 57-65, 2014.

58. Hurtado-Alvarado G, Pavón L, Castillo-García SA, Hernández ME, Domínguez-Salazar E, Velázquez-Moctezuma J and Gómez-González B: Sleep loss as a factor to induce cellular and molecular inflammatory variations. Clin Dev Immunol 2013: 801341, 2015

59. Dantzer R: Cytokine-induced sickness behavior: Where do we stand? Brain Behav Immun 15: 7-24, 2001.

60. Xiang B, Liu K, Yu M, Liang X, Huang C, Zhang J, He W, Lei W, Chen J, Gu X and Gong K: Systematic genetic analyses of GWAS data reveal an association between the immune system and insomnia. Mol Genet Genomic Med 7: e00742, 2019.

61. Trabanco AA and Cid JM: mGluR2 positive allosteric modulators: A patent review (2009-present). Expert Opin Ther Pat 23: 629-647, 2013.

62. Wood CM, Wafford KA, McCarthy AP, Hewes N, Shanks E, Lodge D and Robinson ESJ: Investigating the role of mGluR2 versus mGluR3 in antipsychotic-like effects, sleep-wake architecture and network oscillatory activity using novel Han Wistar rats lacking mGluR2 expression. Neuropharmacology 140: 246-259, 2018.

63. He B, Li Q, Jia Y, Zhao L, Xiao F, Lv C, Xu H, Chen X and Bi K: A UFLC-MS/MS method for simultaneous quantitation of spinosin, mangiferin and ferulic acid in rat plasma: Application to a comparative pharmacokinetic study in normal and insomnic rats. J Mass Spectrom 47: 1333-1340, 2012.

64. Weber F, Chung S, Beier KT, Xu M, Luo L and Dan Y: Control of REM sleep by ventral medulla GABAergic neurons. Nature 526: 435-438, 2015.

65. Hoffmann KM, Beltran L, Ziemba PM, Hatt H and Gisselmann G: Potentiating effect of glabridin from Glycyrrhiza glabra on $\mathrm{GABA}_{\mathrm{A}}$ receptors. Biochem Biophys Rep 6: 197-202, 2016.

66. Mckernan RM, Rosahl TW, Reynolds DS, Sur C, Wafford KA, Atack JR, Farrar S, Myers J, Cook G, Ferris P, et al: Sedative but not anxiolytic properties of benzodiazepines are mediated by the GABA(A) receptor alpha1 subtype. Nat Neurosci 3: 587-592, 2000.

67. Woo JH, Ha TW, Kang JS, Hong JT and Oh KW: Potentiation of decursinol angelate on pentobarbital-induced sleeping behaviors via the activation of GABAA-Ergic systems in rodents. Korean J Physiol Pharmacol 21: 27-36, 2017.

This work is licensed under a Creative Commons Attribution-NonCommercial-NoDerivatives 4.0 International (CC BY-NC-ND 4.0) License. 